\title{
ESTUDO TEÓRICO - EXPERIMENTAL: SUSPENSÃO MAGNÉTICA E AÇÃO MOTORA
}

\author{
Paulo Irineu Koltermann - paulo.koltermann@ufms.br ${ }^{1}$ \\ Valmir Machado Pereira - valmir.pereira@ufms.br ${ }^{l}$ \\ Jéferson Meneguin Ortega-jeferson.ortega@ufms.br ${ }^{l}$ \\ Evandro Mazina Martins - evandro.mazina@ufms.br ${ }^{l}$ \\ EnisVieira-enisvieira@gmail.com ${ }^{2}$ \\ ${ }^{1}$ Professor do Curso de Engenharia Elétrica \\ ${ }^{2}$ Acadêmico do Curso de Engenharia Elétrica \\ Universidade Federal de Mato Grosso do Sul - UFMS \\ Faculdade de Engenharias, Arquitetura e Urbanismo e Geografia-Faeng \\ Av. Costa e Silva $s / n^{o}-$ Bairro Universitário \\ 79070-900 - Campo Grande - Mato Grosso do Sul-MS
}

Resumo: Os dispositivos eletromagnéticos exercem um papel fundamental para o avanço das aplicações tecnológicas. Neste contexto se destaca a importância do ensino de eletromagnetismo nos cursos de Engenharia Elétrica no sentido de dotar os acadêmicos de conhecimentos sólidos neste tema. Este trabalho teve como objetivo demonstrar para os alunos de graduação em Engenharia Elétrica os aspectos fundamentais correlacionados com as Leis de Ampere e de Faraday-Lenz e suas aplicações, bem como interpretar os efeitos envolvidos na operação de circuitos eletromagnéticos. Para tanto foi montado um sistema experimental composto por um eletroímã dotado de um corpo móvel, consistindo de um cilindro de alumínio que atua como objeto repelido que, a partir das forças originadas das correntes induzidas promove a suspensão do mesmo. Esse cilindro sendo colocado no centro geométrico de um conjunto de bobinas alimentadas por sistema trifásico está também sujeito a forças de torque produzidas pelas correntes induzidas pelo campo girante trifásico resultante. Com essas duas situações, demonstra-se o processo de formação do Torque de giro e Força de repulsão por ação das correntes induzidas no cilindro de alumínio (rotor). Os efeitos eletromagnéticos de suspensão magnética e de ação motora são atualmente muito utilizados em trens de alta velocidade, denominados Maglev. Os resultados obtidos além de despertar a curiosidade científica, permitem pela implementação experimental, estimular o espírito empreendedor e criativo dos alunos e proporciona uma maior integração de conhecimentos teórico-práticos e manuseio com materiais e instrumentos de medição elétrica.

Palavras-chave: Eletromagnetismo. Força Magnética. Dispositivo Eletromagnético.

\section{INTRODUÇÃO}

As Diretrizes Curriculares Nacionais - DCN's para os cursos de engenharia recomendam que as práticas de ensino-aprendizagem do professor devem estar focadas para a ampliação de competências e capacidades por meio de técnicas de ensino que incentivem o desenvolvimento da autonomia, aprendizagem ativa e a busca por novas habilidades pelos alunos. Para que esses aprendizados se transformem em oportunidades concretas de sucesso, o processo de transposição de conhecimentos deve unir-se à interdisciplinaridade e à transversalidade (OLIVEIRA, 2019). 
Os estudos aplicados experimentalmente são instrumentos que devem ser utilizados como parte do processo de ensino-aprendizagem, pois é através desse processo que ocorre um maior desenvolvimento na construção de conhecimentos físicos que são mais importantes e significativos, promovendo concomitantemente a construção de capacidades, competências e habilidades novas para os alunos. Configura-se, portanto, o método experimental, como um procedimento que propicia ao ensino da Engenharia maior materialidade e sensibiliza os alunos na busca de soluções técnico-científicas, uma vez que meio delas passam a compreender melhor os fenômenos do mundo (STEPHAN, 2002). Dessa forma, salienta-se a importância do papel do professor, como profissional habilitado, no sentido de despertar a pesquisa através de uma base integrada de conhecimento maior possível e convidar o aluno a se envolver com esse novo saber, utilizando-se da criatividade na montagem desses meios experimentais.

A experimentação é um recurso de múltiplas possibilidades durante a aprendizagem significativa. Além de permitir a vivência prática de algumas teorias e o aprendizado do correto manuseio de instrumentos científicos, contribui para o desenvolvimento da capacidade cognitiva e do raciocínio científico, favorecendo o trabalho cooperativo por meio da discussão, da negociação de atitudes, da divisão de tarefas e da argumentação lógica (GIANI, 2010).

Também, de acordo com Ausubel (2003), a aprendizagem significativa caracteriza-se, por uma interação entre aspectos relevantes da estrutura cognitiva - subsunçores e as novas informações. Os organizadores prévios fazem a ponte entre os subsunçores e o novo conhecimento. Nesse processo, o novo conhecimento é integrado e adquire significado na estrutura cognitiva, de maneira não arbitrária e não literal, contribuindo para diferenciação, elaboração e estabilidade dos subsunçores preexistentes e, consequentemente, da estrutura cognitiva.

Uma importante base teórica desenvolvida, pelo seu papel de integração dos conhecimentos de eletromagnetismo, foi desenvolvida por Maxwell, em 1873, quando ele formulou uma teoria físico-matemática unificada para o eletromagnetismo clássico. As complexas equações de Maxwell influenciariam profundamente a ciência, a tecnologia e a sociedade, como foi constatado nos anos que se seguiram ao desenvolvimento da teoria. (ASSUMPÇÃO BASTOS, 2008)

Dada a complexidade e importância do tema, as equações de Maxwell não são um tema fácil de abordagem, e sua compreensão precisa contar com experimentos lúdicos e simplificações matemáticas, preservando a essência dos ensinamentos.

\section{PRINCÍPIOS BÁSICOS DO ELETROMAGNETISMO - LEIS FUNDAMENTAIS}

As equações de Maxwell (ASSUMPÇÃO BASTOS, 2008) unificaram os diversos estudos de abordagem do eletromagnetismo existentes na época, envolvendo o tempo e o espaço, aplicados às grandezas eletromagnéticas.

$\mathrm{O}$ conjunto de Equações (1) e (2) resume os fenômenos eletromagnéticos ligados à magnetodinâmica, tendo como principal grandeza o campo magnético (indução magnética), originado de corrente elétrica em condutores, que demonstram os fenômenos variáveis no tempo, cujas leis fundamentais correspondem à Lei de Ampère e Faraday-Lenz, acompanhada das relações de passagem:

$$
\mathbf{B}=\mu \mathbf{H} \quad \mathbf{J}=\sigma \mathbf{E}
$$


"Os desafios para formar hoje o engenheiro do amanhã"

$$
\operatorname{rot} \mathbf{H}=\mathbf{J} \quad \operatorname{div} \mathbf{B}=0 \quad \operatorname{rot} \mathbf{E}=-\frac{\partial \mathbf{B}}{\partial t}
$$

A primeira equação indica qualitativamente e quantitativamente a formação de $\mathbf{H}$ a partir da densidade de corrente $\mathbf{J}$. A Equação (3) relembra que sua expressão sob a forma integral é:

$$
\oint_{\mathrm{L}(\mathrm{S})} \mathbf{H} . \mathbf{d l}=\mathrm{I}
$$

que indica que a circulação de $\mathbf{H}$ ao longo de um caminho $\mathrm{L}(\mathrm{S})$ que envolve uma seção $\mathrm{S}$ é igual à corrente atravessando esta seção (equação de circulação de campo).

A equação envolvendo a variação temporal de $\mathrm{B}$ particulariza o domínio da magnetodinâmica, que indica que a variação temporal de $\mathbf{B}$ cria um campo elétrico $\mathbf{E}$ considerando a incidência do mesmo em corpo de material condutor, por ele circulará uma densidade de corrente $\mathbf{J}(\mathbf{J}=\sigma \mathbf{E})$ que terá a propriedade de cria um campo magnético no interior do núcleo. A Equação (4) indica que este campo terá o sentido oposto à variação $\partial \mathbf{B} / \partial \mathrm{t}$, tentando minimizar o aumento de $\mathbf{B}$.

$$
\operatorname{rot} \mathbf{E}=-\frac{\partial \mathbf{B}}{\partial t}
$$

O sentido em que "E" se estabelece foi determinado por Lenz, sendo chamada de "lei de Lenz", cuja essência é resumida na seguinte frase: "O sentido da corrente induzida é tal que o fluxo produzido por esta corrente tende a se opor a variação de fluxo que a originou". (ASSUMPÇÃO BASTOS, 2008).

\section{LEVITAÇÃO ELETRODINÂMICA OU REPULSÃO ELETROMAGNÉTICA}

A maior parte de dispositivos eletromagnéticos tem a função de transformar a energia elétrica em energia mecânica, ou vice-versa. Como exemplos destes dispositivos existe um vasto número de motores e geradores elétricos, e cita-se também inúmeras aplicações de eletroímã (relés, válvulas eletromagnéticas, transdutores diversos, etc.). Nestas estruturas, procura-se criar esforços mecânicos de tal forma que as partes móveis das mesmas produzam trabalho.

A lei cuja expressão permite calcular a força atuando sobre um segmento elementar dl de condutor percorrido por uma corrente $\mathbf{I}$, sob a ação do campo de vetores $\mathbf{B}$, é dada pela Equação (5) que exprime a força magnética sobre um condutor de comprimento dl.

$$
d F=\text { I. } d \mathbf{l} . B
$$

Pela própria noção do produto vetorial, nota-se que a força é perpendicular ao plano formado pelos vetores $\mathbf{d l}$ e $\mathbf{B}$, obedecendo a regra do triedro direto, e seu módulo é dado na equação (6):

$$
\boldsymbol{d F}=\mathbf{I} \cdot \boldsymbol{d l} \cdot \boldsymbol{B} \cdot \operatorname{sen} \theta
$$

sendo $\theta$ o ângulo formado pelos vetores $\mathbf{d} \ell$ e $\mathbf{B}$, conforme a Figura 1. 
(C) COBENCE

"Os desafios para formar hoje o engenheiro do amanhã"
$\mathrm{Ol}$ a $\mathrm{O3}$ de dezembro Evento On-line

Figura 1 - Diagrama Vetorial: Força Magnética.

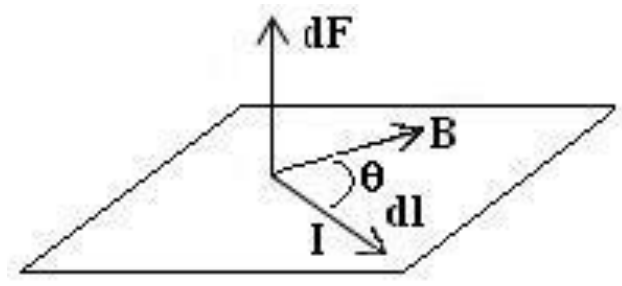

Fonte: Autor

A suspensão magnética é uma forma de levitação magnética na qual existem condutores expostos a campos magnéticos variáveis no tempo. Isso induz correntes parasitas nos condutores que criam um campo magnético repulsivo que mantém os dois objetos separados.

Então, baseado nas equações de Maxwell, mostra-se o mecanismo pelo qual podem aparecer campos magnéticos e elétricos dentro do condutor. (ASSUMPÇÃO BASTOS, 2008). As correntes elétricas $\mathrm{J} c=\sigma \mathrm{E}$, onde $\mathrm{E}$ é o campo dentro do condutor. Para explanação desse efeito, considera-se a placa condutora retangular sujeito às linhas de fluxo do eletroímã, conforme Figura 2.

Figura 2 - Correntes Induzidas - Força de Repulsão.

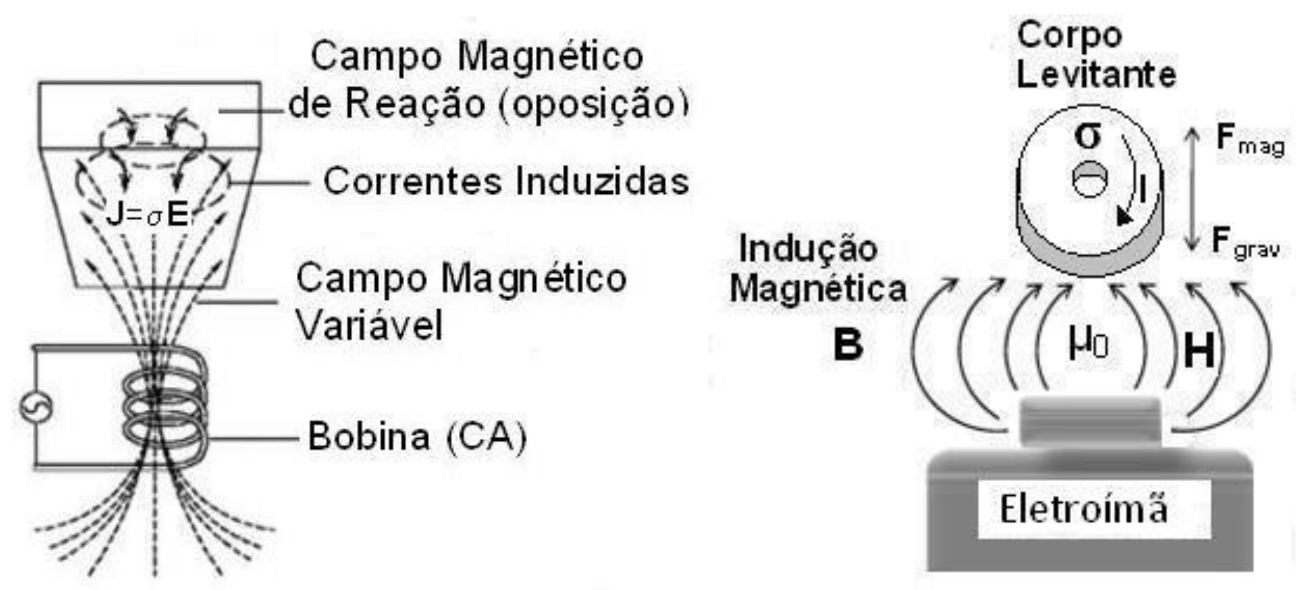

Fonte: Autor 


\section{COBENGE (C) COBENCE 2020 da ABENGE}

\section{"Os desafios para formar hoje o engenheiro do amanhã"}

A Figura 3 apresenta os aspectos construtivos da montagem do sistema de suspensão (eletroímã e cilindro).

Figura 3 - Conjunto Eletroímã - Cilindro.
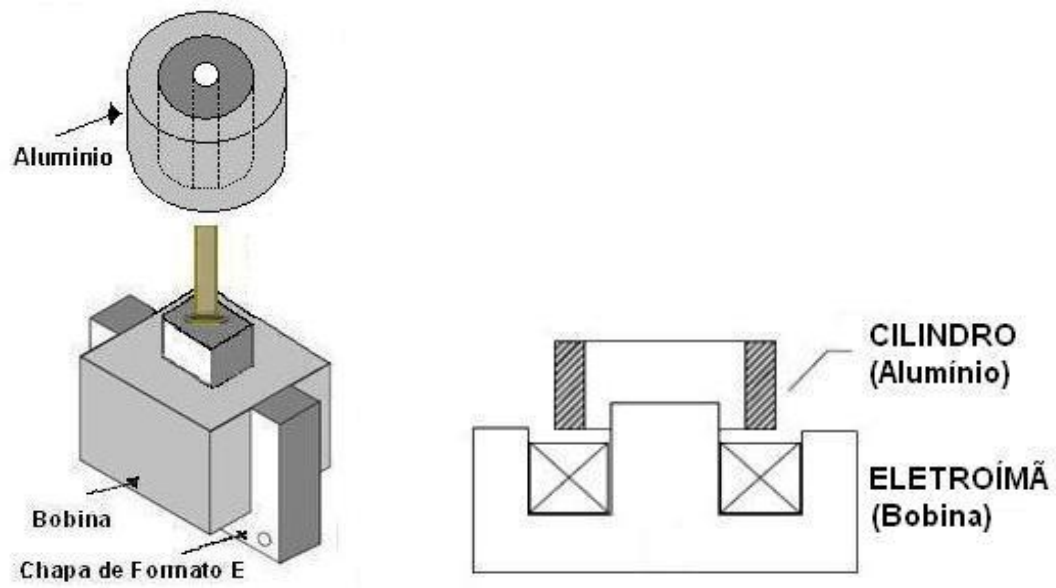

Fonte: Autor

\section{CAMPO GIRANTE - CONJUGADO MOTOR}

Um campo girante trifásico de intensidade constante é normalmente obtido a partir de três bobinas monofásicas fixas defasadas espacialmente de 120 graus. Este princípio do eletromagnetismo clássico é extremamente interessante e sua descoberta possibilitou o desenvolvimento dos modernos motores de corrente alternada, em particular os motores de indução. As bobinas são dispostas espacialmente defasadas de $120^{\circ}$ e alimentadas por fonte elétrica alternada trifásica (defasadas $120^{\circ}$ elétricos e com mesma amplitude), conforme Figura 4.

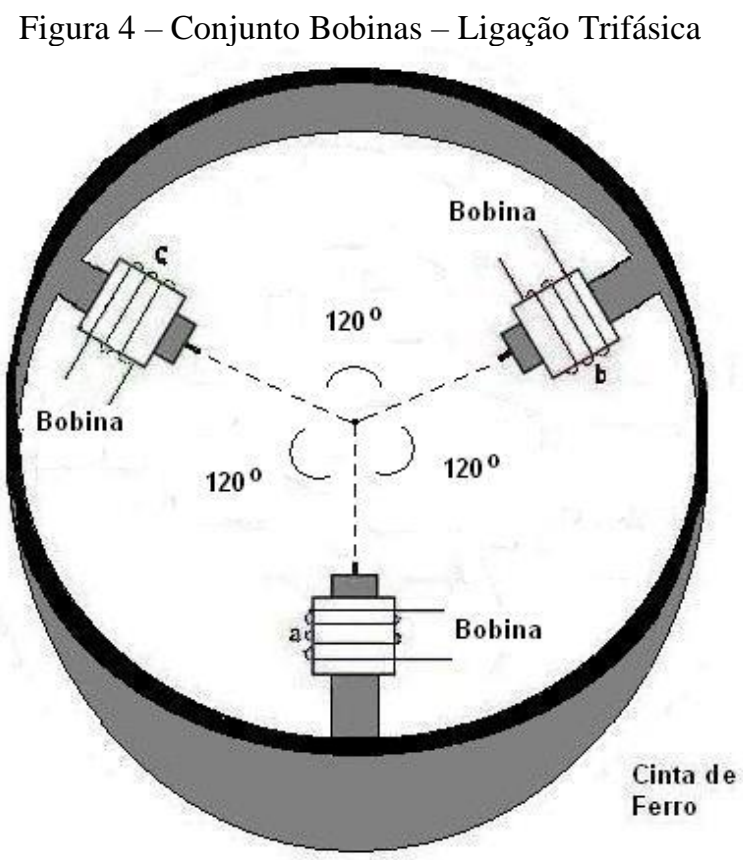

Fonte: Autor 
(C) COBENGE

"Os desafios para formar hoje o engenheiro do amanhã"
$\mathrm{Ol}$ a $\mathrm{O3}$ de dezembro

Evento On-line

Nesse caso será criado um sistema trifásico de correntes $\left(i_{a}, i_{b}, i_{c}\right)$ que gera um sistema trifásico de campos magnéticos $\left(h_{a}, h_{b}, h_{c}\right)$. As respectivas expressões matemáticas para as correntes e os respectivos campos são mostradas nas Equações (7), (8), (9) e (10):

$$
\begin{array}{cl}
\mathrm{i}_{\mathrm{a}}(\mathrm{t})=\mathrm{I}_{\mathrm{am}} \operatorname{sen} \omega \mathrm{t} & \mathrm{h}_{\mathrm{a}}(\mathrm{t})=\mathrm{H}_{\mathrm{am}} \operatorname{sen} \omega \mathrm{t} \\
\mathrm{i}_{\mathrm{b}}(\mathrm{t})=\mathrm{I}_{\mathrm{bm}} \operatorname{sen}\left(\omega \mathrm{t}-120^{\circ}\right) & \mathrm{h}_{\mathrm{b}}(\mathrm{t})=\mathrm{H}_{\mathrm{bm}} \operatorname{sen}\left(\omega \mathrm{t}-120^{\circ}\right) \\
\mathrm{i}_{\mathrm{c}}(\mathrm{t})=\mathrm{I}_{\mathrm{cm}} \operatorname{sen}\left(\omega \mathrm{t}+120^{\circ}\right) & \mathrm{h}_{\mathrm{c}}(\mathrm{t})=\mathrm{H}_{\mathrm{cm}} \operatorname{sen}\left(\omega \mathrm{t}+120^{\circ}\right) \\
\mathrm{I}_{\mathrm{am}}=\mathrm{I}_{\mathrm{bm}}=\mathrm{I}_{\mathrm{cm}}=\mathrm{I}_{\mathrm{m}} \quad \mathrm{H}_{\mathrm{am}}=\mathrm{H}_{\mathrm{bm}}=\mathrm{H}_{\mathrm{cm}}=\mathrm{H}_{\mathrm{m}} \quad \mathrm{H}_{\mathrm{R}}=1,5 \mathrm{H}_{\mathrm{m}}
\end{array}
$$

As três fases a, b e c são deslocadas, uma das outras de $120^{\circ}$, e ligadas uma fonte de alimentação trifásica. A composição vetorial dos três campos magnéticos defasados $120^{\circ}$ elétricos resulta em um campo magnético resultante girante $\mathrm{H}_{\mathrm{R}}$, conforme Figura 5.

Figura 5 - Sistema de Alimentação Trifásico e Campo Girante Resultante.
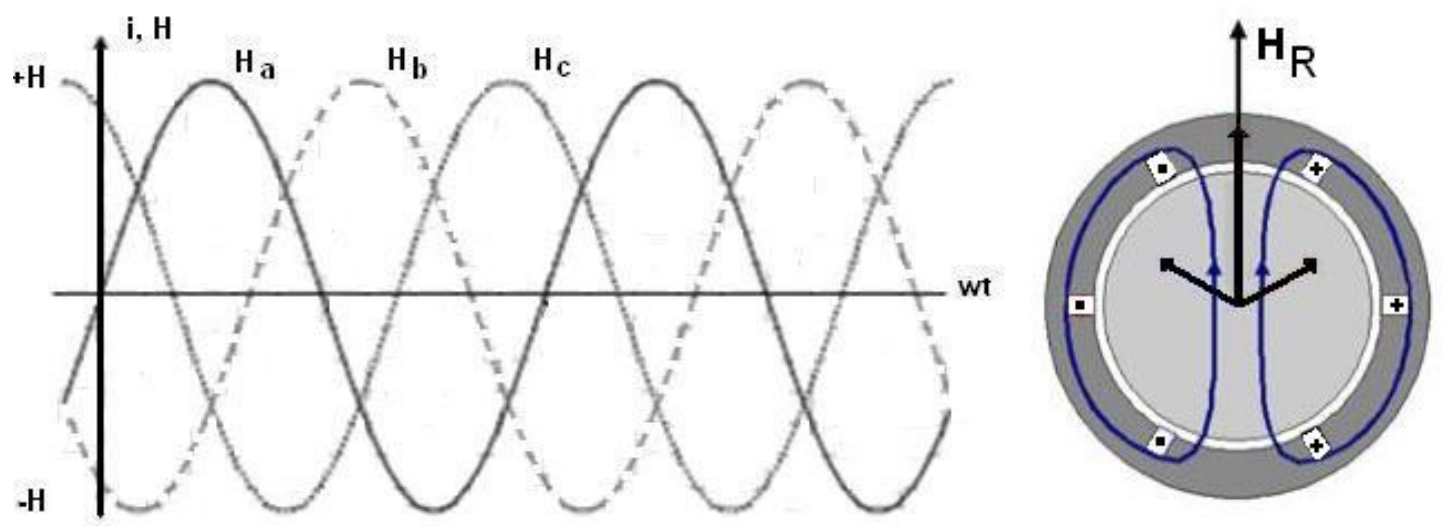

Fonte: Autor

Este campo resultante gira com uma velocidade constante que depende da frequência da fonte e o número de polos obtido da montagem das bobinas. A velocidade de rotação do campo é a velocidade síncrona, cuja expressão é mostrada na Equação (11):

$$
\mathrm{n}=\frac{120 \mathrm{f}}{\mathrm{p}}
$$

Onde:

$\mathrm{n}=$ velocidade em rpm; $\mathrm{f}=$ frequência da rede; $\mathrm{p}=$ número de polos.

O sentido de rotação do campo, que determina o sentido de rotação do motor, depende da sequência das tensões e das ligações das três fases, que na prática poderá ser invertido invertendo as ligações de duas fases quaisquer do estator.

O corpo móvel consiste de material não magnético tipo alumínio (paramagnético), na forma de um cilindro que atuará como rotor, cujo giro se dá em torno de um eixo como mancal radial mecânico - guia de rotação, para fins de estabilidade do corpo girante repelido (suporte do rotor submetido às forças eletromagnéticas de suspensão e torque girantes). 


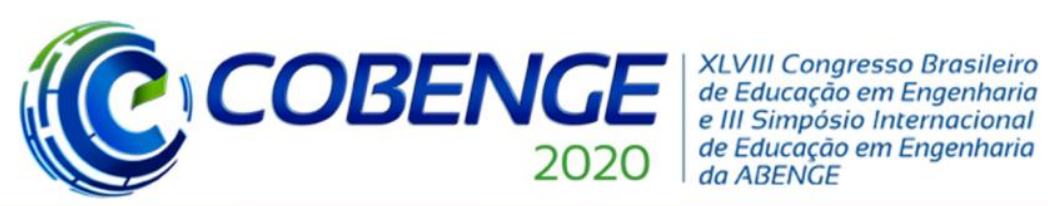

"Os desafios para formar hoje o engenheiro do amanhã"
O1 a 03 de dezembro Evento On-line

Para obtenção da ação motor, a Força $(\mathbf{F}=\mathbf{B} \times \mathbf{\ell . i})$ e, consequentemente o conjugado devem ser proporcionais ao somatório dos produtos das correntes no rotor (cilindro) (i $i_{2}$ ) pela indução magnética (B) apresentada pelo campo rotativo estatórico sobre esses condutores rotóricos, como mostrado na Equação (12).

$$
C=K \cdot B \cdot i_{2}
$$

Ou ainda, que o conjugado deve ser proporcional ao produto entre o valor de pico de "B" (campo girante) provocada pelo estator (conjunto trifásico de bobinas); o valor de pico da onda de FMM provocada pelas correntes rotóricas do cilindro de alumínio e o seno do ângulo entre eles, como mostrado na Equação (13):

$$
C=K \cdot B_{\text {pico }} \cdot F M M_{\text {pico }} \cdot \operatorname{sen} \theta
$$

O ângulo " $\theta$ " é o ângulo entre a indução magnética B (campo girante) e a Força Magnetomotriz (NI) do rotor.

A Figura 6 ilustra os efeitos físicos presentes de suspensão magnética e conjugado motor (torque eletromagnético), demonstrando as Leis de Faraday-Lenz (correntes induzidas) e Força de Lorentz (FITZGERALD, 2006), (CHAPMAN, 2013).

Figura 6 - Conjunto Eletroímã - Corpo Móvel - Ação de Suspensão e Ação Motora
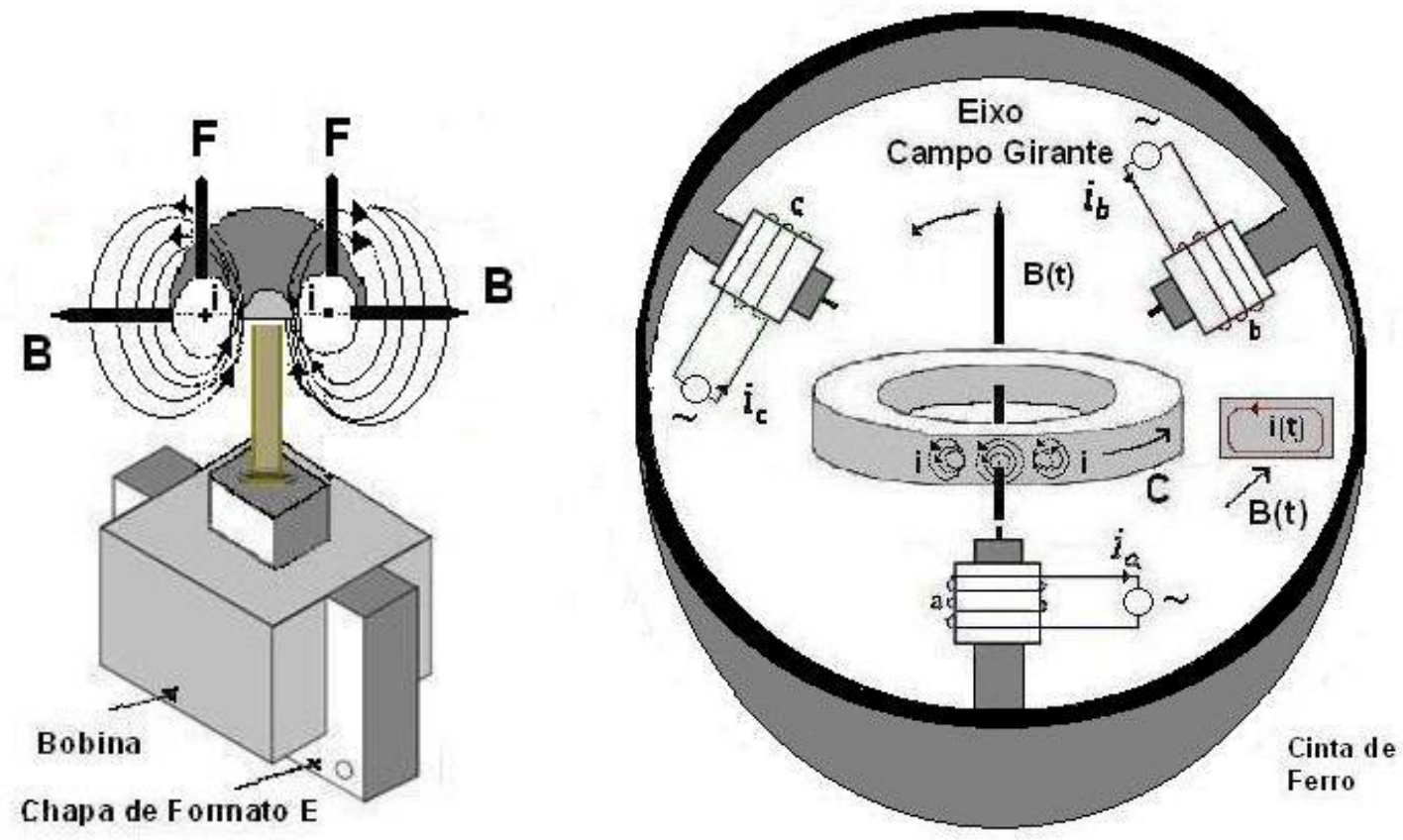

Fonte: Autor

\section{MATERIAIS E MÉTODOS}

\subsection{Materiais utilizados}

O sistema analisado é composto basicamente por dois circuitos elétricos, sendo o primeiro formado pelas três bobinas defasadas espacialmente de $120^{\circ}$, o segundo de um sistema formado por um eletroímã com núcleo ferromagnéticos de chapas tipo E-I acoplado a um eixo com um cilindro-rotor de alumínio que representa o conjunto do sistema de 
suspensão e ação motora. Os dois circuitos elétricos são alimentados por fontes separadas, trifásica e monofásica, respectivamente.

Para a realização do experimento foram utilizados equipamentos, instrumentos e materiais cedidos pelo Laboratório de Máquinas Elétricas Girantes e Transformadores LABMAQ da UFMS.

- Autotransformador Trifásico Variável - VARIAC (0-300 V)

- Autotransformador Monofásico Variável - VARIAC (0-220 V)

- Bobinas (conjunto trifásico) - Bobina (eletroímã)

- Núcleo Magnético - EI-40 - Cilindro de Alumínio

- Voltímetros e Amperímetros

\subsection{Procedimentos Experimentais}

Considerando a estrutura do sistema físico montado foi dada sequência à realização do experimento, efetuando-se as respectivas energizações dos dois circuitos elétricos presentes.

Inicialmente, foi energizada a bobina do eletroímã para promover a levitação do corpo móvel (cilindro de alumínio). A bobina envolta na coluna central do circuito magnético munido de um eixo, é responsável por produzir o campo magnético variável no tempo o qual enlaça o corpo de material condutor cilíndrico disposto sobre o eixo.

As correntes induzidas no cilindro de alumínio produzirão forças repulsoras de origem magnética que se opõem à força peso que age sobre o corpo, promovendo a suspensão do mesmo.

Para a altura da suspensão do cilindro, ponto de operação desejado, a tensão aplicada sobre o eletroímã foi de 70 Volts e a corrente absorvida foi de 6,1 Amperes.

Após concluído esse primeiro processo, o eletroímã é mantido energizado (corpo suspenso) e é energizado o conjunto trifásico de bobinas que será responsável pela produção do campo girante e promoção do conjugado motor no cilindro de alumínio. Tal como ocorre no motor de indução, correntes induzidas surgirão no cilindro de alumínio (rotor) e considerando a presença da indução do campo girante e o efeito do ângulo entre eles, haverá a formação do conjugado motor. Ấ medida que o conjugado motor supera a sua inércia inicial, inicia-se o movimento de giro do cilindro de alumínio (rotor).

Para uma velocidade suportável pelo sistema mecânico (torque eletromagnético), a tensão de linha aplicada sobre o conjunto trifásico de bobinas foi de 165 Volts e a corrente de linha absorvida de 1,2 Amperes.

A mudança do sentido de rotação pode ser feita a partir de mudança da sequência de fases na alimentação do circuito trifásico de bobinas.

Para fins de redução da relutância do ar, o sistema trifásico de bobinas foi interligado por meio de uma cinta de ferro, permitindo assim um caminho mais permeável para o fluxo, reduzindo assim a corrente de excitação absorvida da rede elétrica.

O sistema é apresentado durante as aulas práticas das disciplinas de Circuitos Eletromagnéticos e Máquinas Assíncronas. Durante as demonstrações são suscitadas aos alunos questões referentes aos efeitos físicos presentes e a respectiva correlação com os aspectos teóricos, enfatizando a presença das grandezas vetoriais e a sua interpretação de direção e sentido das mesmas. 


\section{COBENCE de Educação em Engenharia da ABENGE}

"Os desafios para formar hoje o engenheiro do amanhã"

A Figura 7 apresenta a montagem experimental do sistema completo:

Figura 7 - Conjunto Eletroímã - Corpo Móvel - Ação Suspensão e Ação Motora

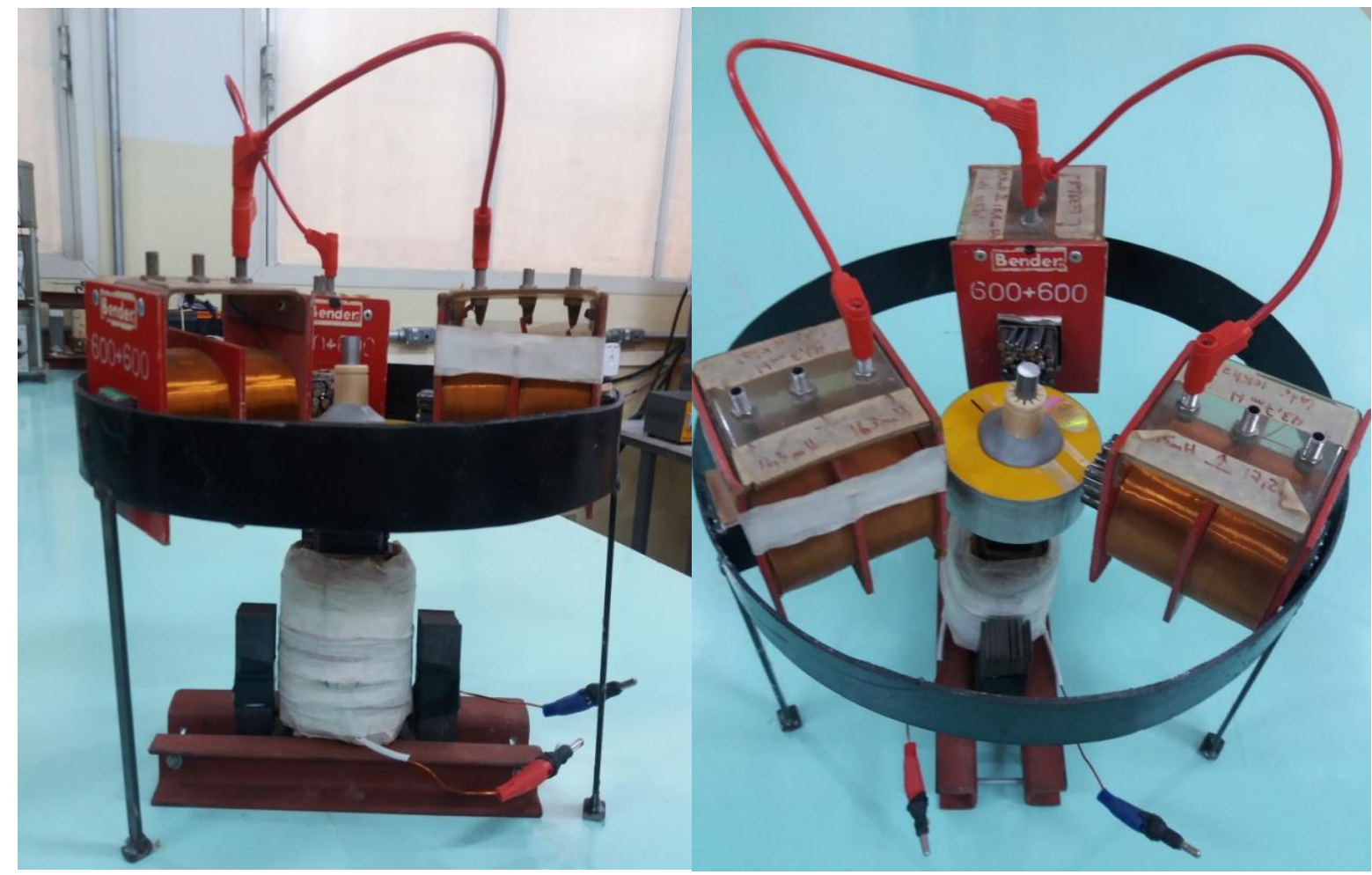

Fonte: Autor

\section{CONSIDERAÇÕES FINAIS}

O trabalho apresenta os conceitos básicos de eletromagnetismo e são abordados efeitos físicos de grande relevância para aplicações tecnológicas. Foi apresentado também o embasamento teórico para o entendimento do experimento (Lei de Faraday-Lenz e Força de Lorentz). A utilização de experimentos é considerada uma eficiente atividade didática em cursos de engenharia, uma vez que concilia teoria e prática, integrando e fomentando novas competências e habilidades. A realização deste trabalho permitiu além de reforçar princípios fundamentais de máquinas elétricas, estimular o desenvolvimento do hábito de pesquisa, a aquisição de novas competências, como instrumentação e interpretação de operação de circuitos eletromagnéticos; o planejamento, em especial no momento em que foi necessário trabalhar a versatilidade do sistema montado. As dificuldades encontradas ao longo do trabalho foram contornadas com criatividade, dedicação e organização do grupo envolvido. Desta forma, pode-se considerar que este sistema físico contempla características pedagógicas relevantes, no que diz respeito ao seu desenvolvimento e ao uso em aulas experimentais no curso de Engenharia Elétrica.

\section{REFERÊNCIAS}

ASSUMPÇÃO BASTOS, João Pedro. Eletromagnetismo para Engenharia: Estática e Quase-Estática. Brasil: Editora UFSC, 2008. 
"Os desafios para formar hoje o engenheiro do amanhã"

AUSUBEL, David. Aquisição e retenção de conhecimentos: uma perspectiva cognitiva. Lisboa: Planalto, 2003.

CHAPMAN, Stephen J. Fundamentos de máquinas elétricas. 5. ed. Porto Alegre (RS): AMGH, 2013.

FREIRE, Paulo. Pedagogia da autonomia: saberes necessários à prática educativa. 43 . ed., São Paulo: Paz e Terra, 2011.

FITZGERALD, A. E., Junior, Charles Kingsley, Umans, Stephen D. - Máquinas Elétricas.

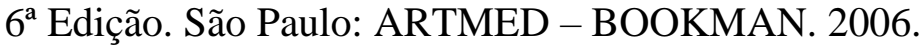

GIANI, Kellen. A experimentação no Ensino de ciências: possibilidades e limites na busca de uma aprendizagem significativa, Dissertação de Mestrado em Ensino de Ciências. Universidade de Brasília, Brasília, 2010.

OLIVEIRA, Vanderli Fava de. A engenharia e as novas DCNs: oportunidades para formar mais e melhores engenheiros / Organizador Vanderli Fava de Oliveira. - 1. ed. - Rio de Janeiro: LTC, 2019.

STEPHAN, Richard, et al. Experiências de Levitação Magnética. Rio de Janeiro In: Congresso Brasileiro de Automática, Anais. UFRJ, p. 309-312, 2002.

\section{THEORETICAL AND EXPERIMENTAL STUDY: MAGNETIC SUSPENSION AND MOTOR ACTION}

Summary: Electromagnetic devices plays a key role for technologic's application advance. Within this context it stands out the relevance of electromagnetism being taught in Electric engineering courses in the sense of providing the academics a solid knowledge in regards to this subject. This work was intended to show Electric Engineer graduating students fundamental aspects correlated to the Ampere and Faraday-Lenz law and it's applications, as well as to interpret the effects involved in electromagnetic circuits operation. Therefore an experimental system composed by an electromagnet equipped of a movable body has been set up consisting of an aluminium cylinder which acts as a repelled object that, due to originated forces by the induced streams it promotes a suspension of itself. This cylinder being placed in the geometrical center of a set of coils fed by three-phase system is also subject to torque forces produced by the induced currents by the synchronous field three-resulting. In both situations, it's noticeable the formation process of Spin Torque and Repulsion Force by action of induced currents in the aluminium cylinder (rotor). The electromagnetic effects of magnetic suspension and motored action are currently very much used in high speed trains, they're denominated Maglev. The obtained results besides bringing up scientific curiosity, it allows though experimental implementation, to stimulate an entrepreneur spirit and manoeuvring with electric measurements instruments and magnetic materials.

Keywords: Electromagnetism. Magnetic Force. Electromagnetic Device. 